\title{
A Web-Based Mental Health Platform for Individuals Seeking Specialized Mental Health Care Services: Multicenter Pragmatic Randomized Controlled Trial
}

Jennifer M Hensel ${ }^{1,2,3,4}$, MSc, MD; James Shaw ${ }^{1,4}$, PhD; Noah M Ivers ${ }^{1,4,5,6}$, MD, PhD; Laura Desveaux ${ }^{1,4,6}$, PhD; Simone N Vigod ${ }^{1,2,4,6}$, MSc, MD; Ashley Cohen ${ }^{7}$, MSc; Nike Onabajo ${ }^{1}$, MSc; Payal Agarwal ${ }^{1,5}$, MD; Geetha Mukerji ${ }^{1,6,8}$, MSc, MD; Rebecca Yang ${ }^{1}$, MPH; Megan Nguyen ${ }^{1}$, MN; Zachary Bouck ${ }^{1}$, MSc; Ivy Wong ${ }^{1}$, MPA, MPAff; Lianne Jeffs $^{7}$, RN, PhD; Trevor Jamieson ${ }^{1,8}$, MD, MBI; R Sacha Bhatia ${ }^{1,4,8}$, MD, MBA

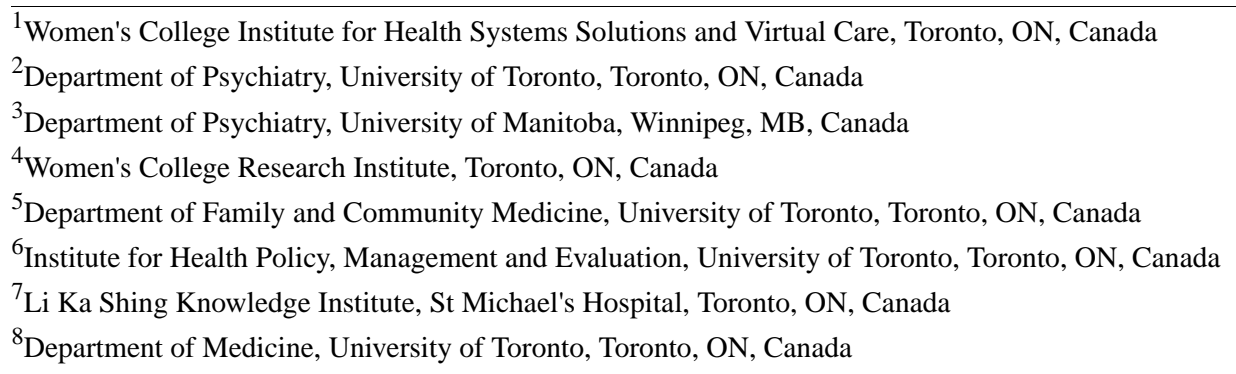

Corresponding Author:

Jennifer M Hensel, MSc, MD

Women's College Institute for Health Systems Solutions and Virtual Care

76 Grenville St

Toronto, ON, M5S 1B2

Canada

Phone: 14163236400 ext 5126

Fax: 14163236004

Email: jennifer.hensel@wchospital.ca

\section{Abstract}

Background: Web-based self-directed mental health applications are rapidly emerging to address health service gaps and unmet needs for information and support.

Objective: The aim of this study was to determine if a multicomponent, moderated Web-based mental health application could benefit individuals with mental health symptoms severe enough to warrant specialized mental health care.

Methods: A multicenter, pragmatic randomized controlled trial was conducted across several outpatient mental health programs affiliated with 3 hospital programs in Ontario, Canada. Individuals referred to or receiving treatment, aged 16 years or older, with access to the internet and an email address, and having the ability to navigate a Web-based mental health application were eligible. A total of 812 participants were randomized 2:1 to receive immediate (immediate treatment group, ITG) or delayed (delayed treatment group, DTG) access for 3 months to the Big White Wall (BWW), a multicomponent Web-based mental health intervention based in the United Kingdom and New Zealand. The primary outcome was the total score on the Recovery Assessment Scale, revised (RAS-r) which measures mental health recovery. Secondary outcomes were total scores on the Patient Health Questionnaire-9 item (PHQ-9), the Generalized Anxiety Disorder Questionnaire-7 item (GAD-7), the EuroQOL 5-dimension quality of life questionnaire (EQ-5D-5L), and the Community Integration Questionnaire. An exploratory analysis examined the association between actual BWW use (categorized into quartiles) and outcomes among study completers.

Results: Intervention participants achieved small, statistically significant increases in adjusted RAS-r score (4.97 points, $95 \%$ CI 2.90 to 7.05$)$, and decreases in PHQ-9 score ( -1.83 points, 95\% CI -2.85 to -0.82$)$ and GAD-7 score ( -1.55 points, $95 \%$ CI -2.42 to -0.70$)$. Follow-up was achieved for 55\% (446/812) at 3 months, $48 \%$ (260/542) of ITG participants and 69\% (186/270) of DTG participants. Only 58\% (312/542) of ITG participants logged on more than once. Some higher BWW user groups had significantly greater improvements in PHQ-9 and GAD-7 relative to the lowest use group. 
Conclusions: The Web-based application may be beneficial; however, many participants did not engage in an ongoing way. This has implications for patient selection and engagement as well as delivery and funding structures for similar Web-based interventions.

Trial Registration: ClinicalTrials.gov NCT02896894; https://clinicaltrials.gov/ct2/show/NCT02896894 (Archived by WebCite at http://www.webcitation.org/78LIpnuRO)

(J Med Internet Res 2019;21(6):e10838) doi: 10.2196/10838

\section{KEYWORDS}

internet; mental health; anxiety; depression

\section{Introduction}

Mental illness is prevalent, with estimates suggesting that upward of 1 billion people worldwide could be affected at a given point in time [1]. In addition, mental and substance use disorders are emerging as a leading cause of disability, accounting for nearly $10 \%$ of global disability-adjusted life years [1]. Access to and use of appropriate and timely mental health services and specialists, however, continues to be a challenge because of limited resources and individual-level factors surrounding treatment seeking [2-4]. E-mental health applications can potentially help to address some of these gaps [5]. A number of Web-based interventions including smartphone apps and Web-based treatment programs for common mental disorders have demonstrated small-to-moderate treatment effect sizes for symptom reduction [6-8], although these interventions are commonly recommended as standalone or preventative treatment options for those with milder symptoms, where benefits have been most apparent. Engagement with self-directed, Web-based interventions has been cited as a challenge owing to a range of user and intervention design factors, with multicomponent interventions potentially enhancing engagement through more user choice, added interactivity, and customization [9,10]. Moreover, the general advancement and adoption of virtual care is often in the absence of rigorous evaluation and adequate planning for sustainability and spread $[11,12]$.

Investments in digital health worldwide have included a substantial emphasis on digital and virtual intervention to promote health [13], and Canada is no exception [14]. As a part of a series of demonstration projects being implemented by leading stakeholders in digital health and telemedicine in Ontario, Canada's most populous province, the Big White Wall (BWW) was selected as a solution with the potential to be adopted for mental health. The BWW [15] is a multicomponent moderated internet-based intervention with peer support that provides anonymity. At the time this trial was conducted, there had been no previous randomized trial evaluating the BWW.

The target goal for the Ontario demonstration projects was chronic disease management and high-needs patient populations, so the sponsors intentionally selected specialized mental health treatment settings for the intervention. This study sought to determine the utility of the BWW as a solution in a Canadian setting, specifically for individuals with mental health symptomatology severe enough to warrant a need for specialized mental health care. We investigated the effectiveness of 3 months of access to the BWW for mental health recovery, as well as symptoms of depression and anxiety, quality of life, and integration with one's community, relative to a usual care control group who received delayed access to the intervention after the study period. We hypothesized that the BWW would increase mental health recovery across a variety of mental health-related needs and conditions .

\section{Methods}

\section{Trial Design}

This study was a multicenter, parallel-arm, pragmatic randomized controlled trial. Participants seeking services at specialized mental health and addiction programs at the participating sites were randomized 2:1 to receive immediate access to the BWW for 3 months (immediate treatment group, ITG) or delayed access after a 3-month control period (delayed treatment group, DTG). The trial protocol has been previously published and is available open access [16]. The trial was sponsored by Ontario Telemedicine Network and Canada Health Infoway, both government-funded organizations. Sponsors specified the recruitment target and study settings, but had no involvement in the study design, procedures, data collection, or analysis.

\section{Ethics}

This study received ethical approval from the research ethics boards at all participating sites. All participants gave informed consent before taking part in the study.

\section{Changes to Trial Design After Commencement}

In response to automated follow-up surveys going to email junk boxes, personalized emails were sent with the survey link embedded. The frequency of contact to remind participants to complete follow-up surveys was increased, and surveys were completed over the phone when possible. To meet sponsor recruitment targets, recruitment was extended early in the study to individuals attending clinics rather than the initial approach targeting those on waitlists or being discharged. Given a large amount of referral between programs, early on in recruitment, it became apparent that participants were commonly on waitlists for specialized clinics while receiving active treatment in other recruitment settings, so this change had little impact on the overall composition of the study sample.

\section{Recruitment of Participants and Baseline Assessment}

Participants were recruited from outpatient mental health programs affiliated with 3 hospitals in Ontario: (1) a public psychiatric hospital in a medium-sized city with satellite 
treatment sites in smaller urban centers; (2) a large community hospital located in another medium-sized city with satellite addictions programs; and (3) a large ambulatory academic hospital located centrally in a large metropolitan area. The aim of the study was to determine the utility of the BWW as a broad reaching multicomponent Web-based intervention that crosses all mental health and addiction-related needs. The BWW contains guided educational and course content applicable to a wide range of mental health needs, and the peer support component offers various opportunities to engage in discussion relevant to one's personal needs. Thus, we recruited from a range of outpatient programs which included: adult mood and anxiety psychiatry programs, a substance use program, an emergency department, an urgent care clinic, youth mood and anxiety programs, mood and anxiety psychotherapy programs, trauma therapy programs, and a borderline personality disorder program. Program coordinators, clinicians, and administrators at each site reviewed referral waitlists and clinic rosters to identify potential participants and refer them to the study coordinator, who reached out by telephone or met the individual at the clinic. Eligible participants were aged 16 years or older, had access to the internet and an email address, were able to read English, and willing and able to access and use a Web-based mental health intervention. There were no exclusion criteria but referring clinicians were asked to consider if person would be able to participate appropriately in Web-based peer interactions. There were no imposed restrictions on the use of concomitant care, including accessing other Web-based interventions that may be like the one under investigation.

All participants provided informed consent and baseline variables either in person or by phone with a study team member. Baseline assessment included sociodemographic data and all outcome measures. Participants were also asked about baseline belief in the treatment credibility and outcome expectancy. Participants were asked to rate their agreement with the author-generated question, "Self-help tools including web-based services and books are helpful for people with mental health problems." Item 4 from the Credibility and Expectancy Questionnaire [17] was adapted for this study, "By the end of the [BWW access period], how much improvement in your symptoms do you think will occur?" Participants were asked to rate their response from $0 \%$ to $100 \%$ with options available in $10 \%$ increments. This single item has been shown to correlate strongly with mental health treatment outcomes such as psychotherapy [18]. Participants were given the option to complete additional symptom, function, and service utilization measures over the phone or by computerized survey. Secure computerized assessments were sent electronically to each participant's email address. All data were entered into a REDCap database [19]. Trial recruitment took place between July 2016 and January 2017.

\section{Participant Safety}

The team at each study site monitored adverse events. A data safety and monitoring board consisting of 3 experts external to the research team reviewed the trial data at 3 and 6 months after recruitment started, specifically examining change in item 9 (suicidal ideation) scores on the Patient Health Questionnaire-9 item (PHQ-9), as well as all serious adverse events reported by the participating sites throughout the trial.

\section{Intervention-The Big White Wall}

The BWW is a multicomponent, self-directed, and moderated Web-based intervention founded in 2007 and hosted in the United Kingdom. Access to the BWW was free for study participants for 3 months (ITG during the 3-month trial period and DTG after the initial 3-month trial period). The developers advocate that the BWW can improve mental health symptoms through increasing social engagement, normalizing experiences, educating, and equipping with skills to manage difficulties. All participants maintain anonymity on the site through a unique nonidentifiable user alias and use is participant dependent [15]. The BWW is monitored $24 / 7$ by Wall Guides who are trained mental health professionals employed by the BWW and based in the United Kingdom and New Zealand. These individuals constantly review user activity and posts to ensure the content is appropriate and sensitive to all users. They will engage with users through instant communication and in the case of identified risk, the Wall Guides can identify the location of the user and direct them to use local crisis services. Contact information for local crisis services was provided on the unique landing page created for the Ontario users. The BWW components include (1) educational material, (2) guided support courses based on principles of cognitive behavioral therapy and behavior change, and (3) text communication posts as either 1:1 with another member or a Wall Guide or open to discussion groups composed of any members of the peer community. The educational materials and guided support courses cover a wide range of mental health related topics including grief, depression, anxiety, smoking cessation, substance use, trauma, among others. Users can also post and comment on bricks-creative self-expressions whereby the user designs a brick which they can place in the digital wall. See Figures 1-4 for screenshots of the BWW components. The BWW reaches out to users through their registration email if there has been a prolonged period of inactivity, encouraging users to $\log$ on. In this study, all participants received an email alias that linked to their personal email and a unique prescription for a BWW account. A follow-up call was made by a study team member 3 days after sending the prescription and again after 2 weeks, if the account had not been activated. Technical support was available from the research team, the BWW, and the Ontario Telemedicine Network. 
Figure 1. The Big White Wall offers self assessments across a range of mental health concerns including depression, anxiety, and substance use.

Assess Yourself

\begin{tabular}{ll}
\hline Tests & My Previous Tests \\
\hline
\end{tabular}

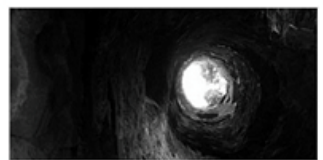

Depression

We can all feel low from time to time. This test will help you look at how | Take Test on Depression low or depressed you may be.

Figure 2. The Useful Stuff pages provide information on mental health conditions and interventions.

\begin{tabular}{|l|}
\hline Useful Stuff \\
\hline Understand more about what' \\
\hline Browse our categories \\
Emotional health \\
\hline Life skills \\
\hline Home and family \\
\hline Relationships \\
\hline Work and study \\
\hline Violence, abuse, and bullying \\
\hline Sex and sexuality \\
\hline Health and lifestyle \\
\hline
\end{tabular}

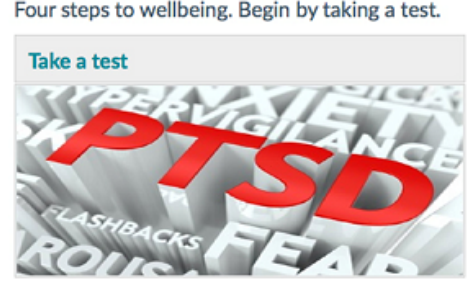

PTSD Checklist - Military

Post-traumatic stress disorder (PTSD) is a condition that may impact persons who have been exposed to traumatic events. If you are.

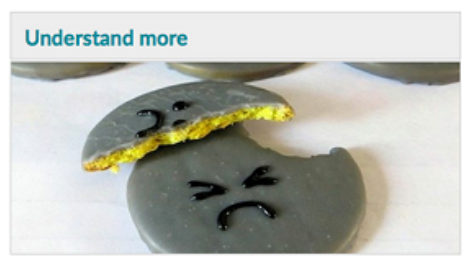

Bake the blues away

The idea that baking is good for the soul has caught the public imagination. The Depressed

Cake Shop is using baking to make life sweeter for.

Figure 3. The Wall is a space for users to post self-expression statements through the creation of artistic bricks. Users can also comment on each other's bricks.

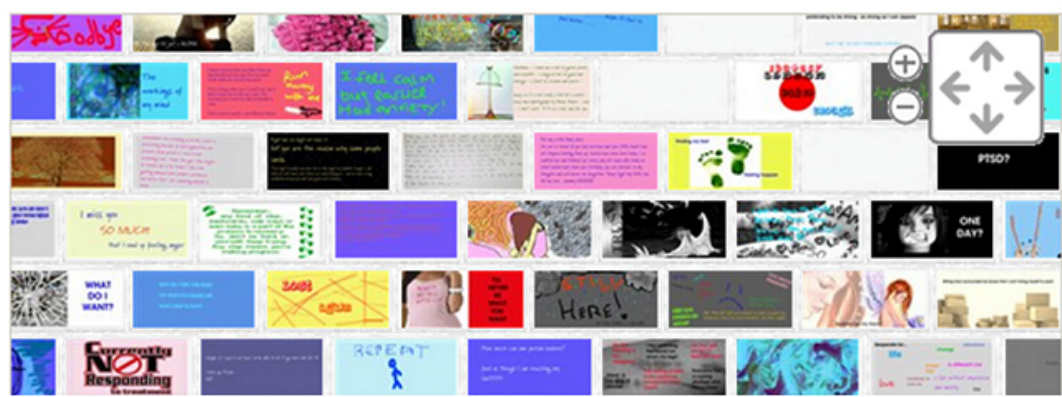

\begin{tabular}{l} 
View recent bricks > \\
View most popular bricks > \\
\begin{tabular}{l} 
Petfriends \\
Abuse wall \\
$\begin{array}{ll}\text { GoodMood } & \text { View wall > }> \\
\text { us joy, comfort, } \\
\text { View wall > }\end{array}$ \\
\hline \\
View wall >
\end{tabular} \\
\hline
\end{tabular}

View recent bricks

Petfriends

us joy, comfort,

sometimes they wall >
View

View all featured walls > 
Figure 4. Moderated personal and group Talkabouts allow users to converse with wall guides and peers regarding their mental health concerns and experiences.

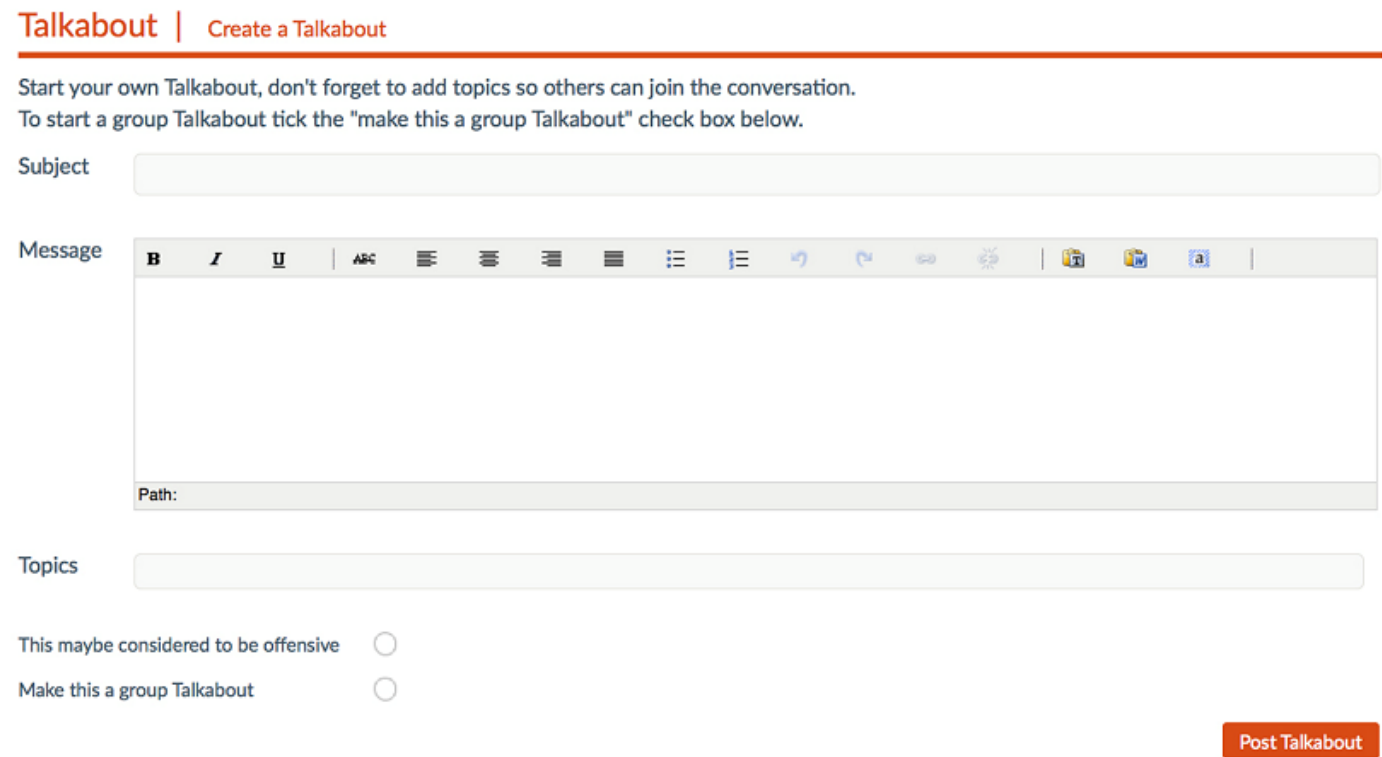

\section{Follow-Up}

Follow-up data were collected between October 2016 and April 2017. All follow-up data were collected by self-report via electronic surveys through REDCap or collected by phone or in person by a study team member and subsequently input into the REDCap database.

Participants received an automated survey link by email 1 week before 3 months postrandomization with a follow-up personalized email containing the survey link. In these emails, DTG participants were reminded that they would receive access to the BWW once the survey was completed, although all participants were ultimately given access regardless of survey completion. At 3 months and 3 months plus 1 week, reminder phone calls were made. Surveys were closed 2 weeks after the 3-month time point. Study team members collecting the outcome assessments were blinded to group allocation. Both baseline and 3-month postrandomization surveys were completed via the Web-based survey in $95 \%$ of cases, with the remainder by phone or in person.

\section{Outcomes}

The primary outcome was mental health recovery at 3 months assessed with the Recovery Assessment Scale-revised (RAS-r). This outcome assesses an individual's orientation toward recovery and self-management across 5 domains: (1) personal confidence and hope, (2) willingness to ask for help, (3) goal and success orientation, (4) reliance on others, and (5) not dominated by symptoms [20]. The study intervention claims to promote self-management specifically, and the RAS-r was chosen as an outcome relevant to participants across all diagnoses. The use of the RAS-r also reflects the current recovery era for mental health policy and services with the focus of treatment shifting to consumers finding satisfying and fulfilling lives, rather than being symptom free [20]. The RAS-r is the most widely used and validated recovery assessment tool and has been studied in several patient populations and shown to correlate with symptoms and function [20]. It is a 24-item scale, with all items scored on a 5-point scale from strongly disagree to strongly agree, with total scores ranging from 24 to 120 .

Secondary outcomes were the PHQ-9 to assess symptoms of depression, Generalized Anxiety Disorder Questionnaire-7 item (GAD-7) to assess symptoms of anxiety, EuroQOL 5-dimension quality of life questionnaire (EQ-5D-5L) to assess quality of life, and the Community Integration Questionnaire (CIQ) [21]. The PHQ-9 scale contains 9 items rated on a 4-point Likert scale from never to almost every day, with a higher score representing more symptoms [22]. The GAD-7 scale has 7 items rated on a 4-point Likert scale from never to almost every day, with a higher score representing a higher likelihood of an anxiety disorder. The EQ-5D-5L questionnaire from the EuroQOL group [23] comprises 5 dimensions: mobility, self-care, usual activities, pain or discomfort, and anxiety/depression, which are rated on 5 levels and can be interpreted as an index score by comparing with available normative values for adult populations. The EQ-5D-5L is paired with a visual analog scale (VAS) rated from 0 to 100 to assess perceived overall health at the time of survey completion. The CIQ consists of 15 items and is intended as a brief, reliable measure of a person's level of integration into the home and community. The overall score can range from 0 to 29 with a higher score indicating better integration [24].

Actual BWW utilization data were obtained for all ITG participants including activation of account and number of logins.

\section{Sample Size}

The target sample size for our trial was set by the sponsors at 1000 participants. We aimed to recruit this number of participants, with an expectation that loss to follow-up would be approximately $30 \%$ based on data from other similar trials [25]. We calculated the minimal detectable difference between the 2 treatment groups for a linear regression analysis controlling 
for baseline score assuming a $0 \cdot 8$ correlation between baseline and 3-month follow-up RAS-r measurements. Assuming 30\% loss to follow-up, for a sample size of 700 after attrition, allocated in a 2:1 ratio, using an alpha of .05 and power of 0.9 , the minimal detectable difference was 1.35 on the RAS-r.

\section{Randomization, Concealment, and Blinding}

Participants were randomized 2:1 to the ITG and DTG groups. A 2:1 randomization ratio was used to offer the intervention immediately to a higher number of participants and increase recruitment. Randomization sequences, using block sizes of 3 or 6 were computer generated by an organization external to the research team, with stratification by site and recruitment setting. Group allocation sequence was concealed but once allocated, participants were not blinded. Participants allocated to the DTG group received a telephone or email notification that they would gain access to the website after a 3-month delay. Most follow-up data were collected by Web-based survey, but in the case where follow-up data were collected by an assessor, the assessor was not the same as the person who did the initial data collection, as this person also disclosed randomization to the participant and would not be blinded at follow-up. Data analysts remained blinded throughout the study.

\section{Statistical Analysis}

The primary outcome, RAS-r, was analyzed at 3 months for all participants regardless of whether they activated their BWW account, but individuals with missing data at 3 months were excluded. We planned to repeat the analysis using a marginal structural model to account for attrition; however, owing to higher than expected attrition that was nonrandom, this analysis was not completed. RAS-r scores at 3 months were modelled with a linear regression controlling for baseline RAS-r score and treatment group. A second analysis controlled for prespecified covariates including baseline PHQ-9, baseline GAD-7, age, gender, recruitment setting, and age of first onset of mental health problems. The same analysis was repeated for all secondary outcomes at 3 months. Analyses were completed after data collection was complete. BWW utilization data were analyzed descriptively to illustrate uptake of the intervention in the ITG participants only.

\section{Exploratory Analysis}

Among ITG participants who completed the 3-month outcome measures, we examined whether there was an association between actual utilization of the application and the primary and secondary outcomes. Utilization of the application was defined by number of BWW logins, with users categorized into 4 groups based on distribution quartiles: 0 to 1 login, 2 to 3 logins, 4 to 9 logins, and 10 or more logins. Owing to degree of missingness, primary and secondary outcomes were separately modeled with repeated measures ANOVA using time as a repeated measure. We tested for an interaction between BWW use and time, after adjusting for same covariates as in the main analysis. Significant main effects were explored with post-hoc Bonferroni tests adjusted for multiple comparisons.

\section{Results}

We approached 1455 individuals, of whom 975 (67.0\%) consented. Of the $975,163(16.7 \%)$ did not complete the baseline assessment questionnaire, leaving 812 to be randomized, $270(33.3 \%)$ to DTG and $542(67.7 \%)$ to ITG (see Figure 5). Distribution of participants across recruitment settings was as follows: adult mood and anxiety psychiatry programs $(\mathrm{n}=294 / 812,36.2 \%)$, youth $\operatorname{mood}$ and anxiety programs $(\mathrm{n}=42 / 812,5 \%)$, mood and anxiety psychotherapy programs $(\mathrm{n}=73 / 812,9 \%)$, emergency department/urgent care clinic $(\mathrm{n}=139 / 812,17.1 \%)$, borderline personality/trauma therapy programs $(\mathrm{n}=114 / 812,14.0 \%)$, and substance use program $(\mathrm{n}=150 / 812,18.5 \%)$. Follow-up was achieved for $446 / 812$ $(54.9 \%)$ at 3 months, $260 / 542(48.0 \%)$ in the ITG group and $186 / 270(68.9 \%)$ in the DTG. At baseline, the randomized groups were well-balanced in terms of sociodemographic, mental health variables, and previous 3-month health care utilization (Table 1).

Figure 5. CONSORT flow diagram of participants through the trial. BWW: Big White Wall.

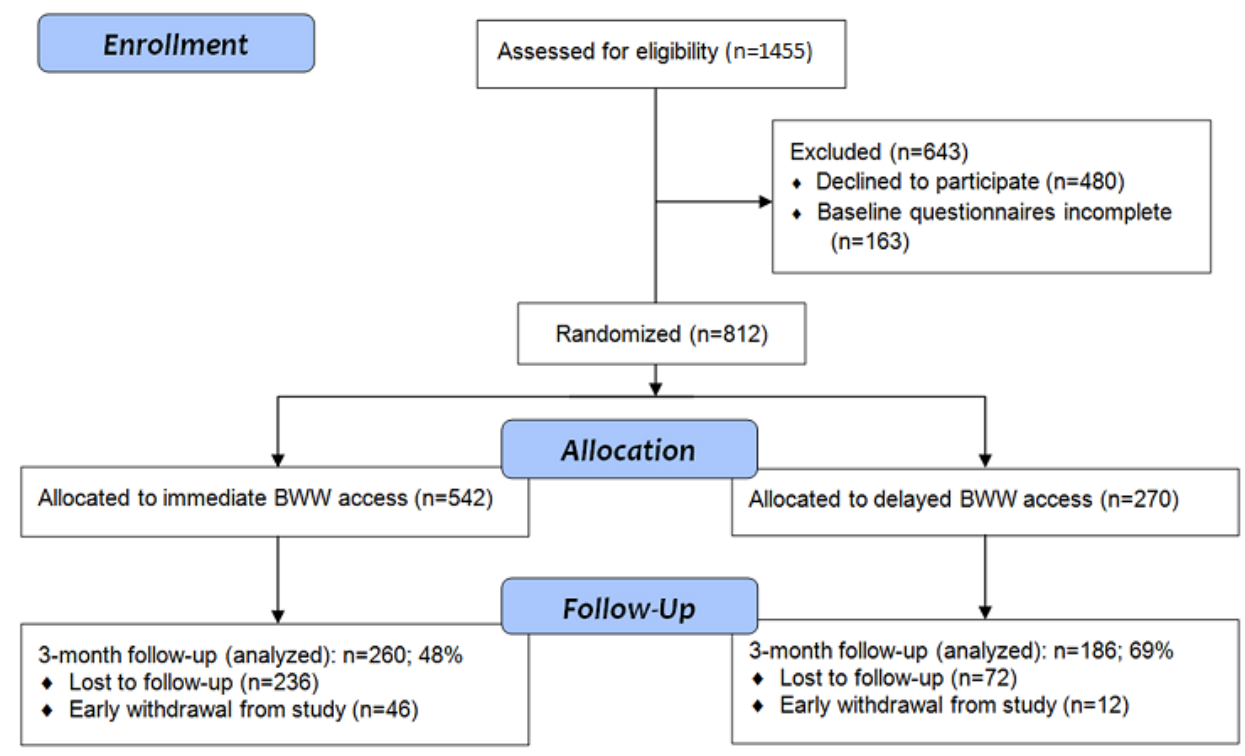


Table 1. Baseline characteristics of study participants by group.

\begin{tabular}{|c|c|c|}
\hline Variables & Immediate Treatment Group $(\mathrm{n}=542)^{\mathrm{a}}$ & Delayed Treatment Group $(\mathrm{n}=270)^{\mathrm{a}}$ \\
\hline Age (years), mean (SD) & $41.5(13.4)$ & $40.0(13.9)$ \\
\hline \multicolumn{3}{|l|}{ Gender, n (\%) } \\
\hline Male & $143(27)$ & $59(22)$ \\
\hline Female & $391(73)$ & $207(78)$ \\
\hline Transgendered & $1(0)$ & $1(0)$ \\
\hline \multicolumn{3}{|l|}{ Ethnicity, n (\%) } \\
\hline White & $440(82)$ & $219(83)$ \\
\hline Non-white & $98(17)$ & $46(18)$ \\
\hline \multicolumn{3}{|l|}{ Relationship status, n (\%) } \\
\hline In a relationship & $286(53)$ & $127(47)$ \\
\hline Not in a relationship & $252(47)$ & $142(52)$ \\
\hline \multicolumn{3}{|l|}{ Employment status, $\mathbf{n}(\%)$} \\
\hline Full-time (including homemaker with young children) & $181(33)$ & $96(35)$ \\
\hline Part-time/volunteer/homemaker without young children & $100(19)$ & $41(15)$ \\
\hline Not working (retired owing to age or actively looking for work) & $33(13)$ & $26(14)$ \\
\hline Not working (not looking for work) & $84(35)$ & $57(31)$ \\
\hline \multicolumn{3}{|l|}{ Household income in Can \$, $n(\%)$} \\
\hline$<35 \mathrm{~K}$ & $238(48)$ & $126(52)$ \\
\hline $35 \mathrm{~K}-50 \mathrm{~K}$ & $56(11)$ & $38(16)$ \\
\hline $50 \mathrm{~K}-80 \mathrm{~K}$ & $82(16)$ & $25(10)$ \\
\hline$>80 \mathrm{~K}$ & $123(25)$ & $52(22)$ \\
\hline Age first experienced mental health problems (years), mean (SD) & $18.7(12.5)$ & $19.0(12.7)$ \\
\hline Age first sought help (years), mean (SD) & $26.7(12.9)$ & $26.3(13.0)$ \\
\hline Taking medication at baseline, $\mathrm{n}(\%)$ & $438(81)$ & $204(76)$ \\
\hline Previous 3-month hospitalization, n (\%) & $47(9)$ & $23(9)$ \\
\hline Previous 3-month emergency room visit, $\mathrm{n}(\%)$ & $77(14)$ & $27(10)$ \\
\hline \multicolumn{3}{|c|}{ Agree with: Self-help tools helpful for people with mental health problems, $n(\%)$} \\
\hline Definitely agree & $203(38)$ & $110(41)$ \\
\hline Somewhat agree & $310(58)$ & $151(56)$ \\
\hline Somewhat or completely disagree & $24(4)$ & $8(3)$ \\
\hline \multicolumn{3}{|c|}{ How much expected improvement in mental health through $B W W^{b, c}, n(\%)$} \\
\hline Less than $50 \%$ & $194(38)$ & $110(42)$ \\
\hline $50 \%$ & $141(26)$ & $76(29)$ \\
\hline More than $50 \%$ & $179(35)$ & $76(29)$ \\
\hline
\end{tabular}

${ }^{\mathrm{a}}$ Percentages calculated after missing data removed.

${ }^{\mathrm{b}}$ Responses were recorded in $10 \%$ increments but based on their distribution have been recategorized.

${ }^{\mathrm{c}} \mathrm{BWW}$ : Big White Wall.

There were some differences between those who were lost to follow-up and those who completed the 3-month survey overall and in each group (see Multimedia Appendices 1 and 2). Overall, the survey completers were older, and more likely to be working full-time or unemployed and not looking for work. ITG survey completers were more likely to activate their BWW

accounts and have more logins than noncompleters in that group, with the same age and employment patterns as overall. In the DTG group, the only significant difference found was for recruitment setting; those from youth and emergency or urgent care settings were most likely to complete follow-up. A proportion of survey noncompleters from both the ITG and 
DTG groups withdrew early from the study (46 out of 282 ITG survey noncompleters $(16 \%)$ and 12 out of 84 (14\%) DTG survey noncompleters).

\section{Primary Outcome}

The primary analysis showed a statistically significant difference between the ITG and DTG groups for the RAS-r at 3 months, with the ITG participants having an RAS-r score on average 5.34 points higher than the DTG participants at 3 months (Table 2 ). In the adjusted model, the effect remained significant but slightly lower in magnitude (Table 2).

Table 2. Baseline and 3-month primary and secondary outcomes among survey completers with linear regression results for outcomes at 3 months.

\begin{tabular}{|c|c|c|c|c|c|c|}
\hline \multirow[t]{2}{*}{ Outcome } & \multicolumn{2}{|c|}{$\begin{array}{l}\text { Immediate treatment group } \\
(\mathrm{n}=260) \text {, mean }(\mathrm{SD})\end{array}$} & \multicolumn{2}{|c|}{$\begin{array}{l}\text { Delayed treatment group } \\
(\mathrm{n}=186), \text { mean }(\mathrm{SD})\end{array}$} & \multirow{2}{*}{$\begin{array}{l}\text { Unadjusted treat- } \\
\text { ment effect size } \\
(95 \% \mathrm{CI})^{\mathrm{a}}\end{array}$} & \multirow{2}{*}{$\begin{array}{l}\text { Adjusted treatment } \\
\text { effect size (95\% } \\
\mathrm{CI})^{\mathrm{a}, \mathrm{b}}\end{array}$} \\
\hline & Baseline & 3 months & Baseline & 3 months & & \\
\hline \multicolumn{7}{|l|}{ Primary outcome } \\
\hline Recovery Assessment Scale, revised & $77.4(14.0)$ & $83.3(15.1)$ & $76.3(14.1)$ & $77.2(14.6)$ & $5.32(3.33$ to 7.31$)$ & $4.97(2.90$ to 7.05$)$ \\
\hline \multicolumn{7}{|l|}{ Secondary outcomes } \\
\hline Patient Health Questionnaire-9 item & $14.8(6.9)$ & $11.5(6.4)$ & $16.0(6.5)$ & $14.2(6.8)$ & $\begin{array}{l}-1.95(-2.94 \text { to } \\
-0.95)\end{array}$ & $\begin{array}{l}-1.83(-2 \cdot 85 \text { to } \\
-0 \cdot 82)\end{array}$ \\
\hline $\begin{array}{l}\text { Generalized Anxiety Disorder } \\
\text { Questionnaire-7 item }\end{array}$ & $11 \cdot 5(5 \cdot 6)$ & $9 \cdot 1(5 \cdot 3)$ & $12 \cdot 3(5 \cdot 6)$ & $11 \cdot 4(5 \cdot 7)$ & $\begin{array}{l}-1.75(-2.60 \text { to } \\
-0.90)\end{array}$ & $\begin{array}{l}-1.56(-2.42 \text { to } \\
-0.70)\end{array}$ \\
\hline $\begin{array}{l}\text { EuroQOL 5-dimension quality of } \\
\text { life questionnaire }\end{array}$ & $0.68(0.16)$ & $0.71(0.17)$ & $0.68(0.16)$ & $0.69(0.15)$ & $\begin{array}{l}0.014(-0.009 \text { to } \\
0.036)\end{array}$ & $\begin{array}{l}0.008(-0.015 \text { to } \\
0.031)\end{array}$ \\
\hline EuroQOL Visual Analog Scale & $56.8(19.2)$ & $58.8(21.5)$ & $55.1(19.8)$ & $55.4(21.9)$ & $\begin{array}{l}2.55(-1.17 \text { to } \\
6.26)\end{array}$ & $\begin{array}{l}1.68(-1.97 \text { to } \\
5.32)\end{array}$ \\
\hline $\begin{array}{l}\text { Community Integration Question- } \\
\text { naire }\end{array}$ & $16.9(5.0)$ & $17.0(5.2)$ & $16.7(4.6)$ & $17.1(4.8)$ & $\begin{array}{l}-0.32(-0.93 \text { to } \\
0.29)\end{array}$ & $\begin{array}{l}-0.30(-0.93 \text { to } \\
0.33)\end{array}$ \\
\hline
\end{tabular}

${ }^{\mathrm{a}}$ Delayed treatment group is the reference group in all analyses; all models include baseline score.

${ }^{\mathrm{b}}$ Adjusted for age, sex, recruitment setting, baseline PHQ-9 score, baseline GAD-7 score, and age of first onset of mental health problems.

\section{Secondary Outcomes}

PHQ-9 and GAD-7 scores were significantly lower in the ITG group compared with the DTG group in the main and adjusted analyses (Table 2). No statistically significant differences were found for EQ-5D-5L index score, EQ-VAS, or CIQ (Table 2).

\section{Participant Safety}

The data safety monitoring board reviewed data at 3 and 6 months after the start of recruitment and did not find any substantial increase in suicidal ideation between groups to warrant investigation or early termination. Over the course of the study, one death was reported to the study team and deemed unrelated to the intervention.

\section{Uptake, Use, and Satisfaction With the Intervention}

\section{Big White Wall Activation}

Among the 542 participants who were randomized to receive immediate access to the BWW, 76 (14\%) never activated their BWW account during the study period. Half of these individuals $(n=39,51 \%)$ withdrew early from the study. Reasons for early withdrawal included the following: technical issues, loss of perceived need or interest, and lack of time owing to competing priorities.

\section{Utilization}

There was large variability in the range of total number of times that participants logged on to the site, from 0 to 236 times. The mean number of logins was 8.7 , with a standard deviation of 18.1 , a median of 2 , and a mode of 1 . Only $58 \%(312 / 542)$ of participants logged on 2 or more times, with approximately $20 \%$ of all participants accounting for $80 \%$ of the total logins.

\section{Exploratory Analysis}

There was no significant interaction between BWW use and time for the primary outcome of RAS-r. The interaction was significant for PHQ-9 $\left(F_{3,257}=4.14 ; P=.007\right)$ and GAD-7 $\left(F_{3,267}=3.89 ; P=.009\right)$. In post-hoc analysis for PHQ-9, a significantly greater reduction in score over time was present for the groups with 10 or more logins and 2 to 3 logins, relative to the 0 to 1 login group (4.14 points vs $1.43, P=.03$ and 5.00 vs $1.43, P=.02$, respectively). In post-hoc analysis for GAD-7, only the group with 2 to 3 logins had a significantly greater reduction in score over time compared with the group with 0 to 1 login (4.36 points vs .91 points, $P=.006)$. There was no significant effect of the interaction between BWW use and time on the other secondary outcomes.

\section{Discussion}

\section{Principal Findings}

Immediate access to the BWW resulted in small, significant improvements in mental health recovery, as well as depressive and anxiety symptoms at 3 months compared with those randomized to delayed access. These statically significant findings are limited by high, differential drop out between 
treatment groups and overall, and were below minimal clinically important differences for these outcome measures (eg, a difference of 1.8 on the PHQ-9 where a clinically important change is 5 points) [22]. Engagement with the intervention varied highly and interestingly, we observed the commonly found Pareto principle for population effects-also known as the $80 / 20$ rule [26]. That is, $20 \%$ of users accounted for approximately $80 \%$ of the activity. We found some evidence of a user effect whereby those who completed follow-up and engaged with the application were more likely to experience improvements in symptoms of depression and anxiety. This was not linear, however, with the user group having 2 to 3 logins experiencing the most consistent improvements relative to the lowest user group. To our knowledge, this is the first randomized evaluation of the BWW, and one of a few large multicenter pragmatic trials of any multicomponent internet-based mental health intervention. The study population represents more treatment-refractory and severely symptomatic individuals than are usually included in studies of Web-based mental health applications.

\section{Comparison With Other Studies}

Although the BWW is unique in comparison with other studied applications, the observed low engagement is similar [25]. A comparatively large pragmatic trial that examined 2 computerized modular CBT programs with added telephone support reported that fewer than $20 \%$ of participants had completed all modules at follow-up [25]. Modular-style courses are only one component of the BWW, the other components being peer support, artistic self-expression, and more general psychoeducation. Compared with a modular program, it is more difficult to define an adequate dose for multicomponent interventions such as the BWW [13]. We examined different self-directed doses of the BWW based on logins and did not find a linear relationship between higher use and better outcomes, although it appeared that some engagement may have been better than none. Some users may benefit from a few targeted uses to get direction or motivation. A systematic review examining the relationship between e-therapy adherence and outcomes reported that studies targeting depression did not find a significant impact of total number of logins on outcomes in contrast to studies examining physical health outcomes like weight management or smoking [9]. Studies using modular interventions, however, did report that module completion led to better outcomes. Conversely, one of the main features of the BWW used more often by the higher engagers is its moderated virtual community of peers [15], an intervention for which the evidence has yielded some very mixed results $[27,28]$. Some authors have described a potential harm through aggravated symptoms and what Takahashi et al termed the downward depressive spiral which was linked to higher depressive tendencies at baseline [29]. This may have tempered the improvements in the higher engager groups or conversely may have led to early disengagement from the application as a result of negative reactions to the peer community.

\section{Limitations}

This study experienced loss to follow-up that was proportionally higher in the intervention group. High drop out of up to $50 \%$ in trials of Web-based mental health interventions has been reported [10] and retention in digitized trials has specifically been discussed as a challenge [30]. In our study, the issues encountered with our automated survey were addressed quickly but may have impacted survey completion. More specifically, other studies of Web-based mental health programs have also reported disproportionate dropout in the intervention group [10]. This trend likely represents some study disengagement for intervention-specific reasons such as dislike of the intervention format or lack of perceived utility. In this study, some very early technology issues with BWW activation likely affected early user engagement and the Web-based platform did not include a mobile version of the site or application which could have deterred use for some. To partially address this limitation, we conducted the exploratory analysis on study completers.

We chose the pan-diagnostic RAS-r as the primary outcome, given that our study sample was recruited from a range of mental health settings and participants had a range of mental health needs. The promise of internet-based mental health interventions specifically to support recovery has been discussed [31]; however, this outcome lacks established clinically important cutoffs. Secondary outcomes may have been affected by the lack of diagnostic specificity relative to other studies that have focused on specific diagnoses such as depression and anxiety [6]. In this study, we evaluated the BWW as a solution for any mental health or addiction-related need; although we adjusted for recruitment program, it is possible that the BWW may work better for certain diagnostic or need subgroups, which represents an area for further evaluation.

\section{Conclusions and Policy Implications}

From this trial, we cannot definitively conclude the effectiveness of this or similar solutions at a population level for individuals accessing specialized mental health care. However, internet-based interventions built on evidence-based principles of mental health care are likely to be beneficial where users are motivated to engage and where the format of the application is a good fit. Determining this subset and/or how to effectively motivate more people to engage with the interventions is a critical next step. Pragmatic studies that build in process evaluations and subset analyses that examine moderators of use and related outcomes are required. This study was undertaken as part of a large provincial implementation program with multiple research, clinical, and policy stakeholders involved throughout, a type of integrated, real-world approach to evaluation that is essential to establish effective health systems solutions [32].

\section{Acknowledgments}

The research team would like to acknowledge the site leads, project coordinators, and research assistants for their roles in project planning, recruitment, and data collection: Beth Brannon, Anusha Baskaran, and Alifa Siddiqui (Ontario Shores Centre for Mental Health Sciences); Paul McGary, Tom McHugh, and Joanne Gourgouvelis (Lakeridge Health); Joanne Leung (Women’s College 
Hospital). This study was sponsored by the Ontario Telemedicine Network through funding from Canada Health Infoway and the Ontario Ministry of Health and Long Term Care.

\section{Authors' Contributions}

All authors contributed to the conceptualization of the study and development of the study protocol. Data analyses for all trial outcomes were completed by AC. Additional analyses on BWW utilization data were completed by MN, NO, and JMH. The manuscript was drafted by JMH and revised critically by all authors. All authors have approved the submitted version of the manuscript. JMH acts as the guarantor for this work and affirms that the manuscript is an honest, accurate, and transparent account of the study being reported. Authors had full access to all study data (including statistical reports and tables) and take responsibility for the integrity of the data and the accuracy of the data analysis. Participant-level data are available from the corresponding author upon request.

\section{Conflicts of Interest}

PA consults with and receives personal fees from the study sponsor on projects unrelated to this study. All other authors declare no competing interests that could influence the submitted work. The sponsor was engaged in the selection of the technology vendor, conducted the implementation of the intervention at the sites, provided input into the study design, and funded the study completion. The sponsor was not involved in the data analysis or writing of the manuscript. The sponsor reviewed the manuscript for accuracy or reporting related to the sponsor involvement. The vendor (BWW) was not involved in the study design, development, implementation, analysis, or writing of this manuscript. The researchers acted independently of all study sponsors and the vendor.

\section{Multimedia Appendix 1}

Baseline characteristics of study participants who did and didn't complete the follow-up measures at 3 months.

[PDF File (Adobe PDF File), 90KB-Multimedia Appendix 1]

\section{Multimedia Appendix 2}

Baseline characteristics of study participants by group, who did and didn't complete the follow-up measures at 3 months.

[PDF File (Adobe PDF File), 63KB-Multimedia Appendix 2]

\section{Multimedia Appendix 3 \\ CONSORT-eHEALTH checklist (V 1.6.1). \\ [PDF File (Adobe PDF File), 2MB-Multimedia Appendix 3]}

\section{References}

1. Whiteford HA, Ferrari AJ, Degenhardt L, Feigin V, Vos T. The global burden of mental, neurological and substance use disorders: an analysis from the Global Burden of Disease Study 2010. PLoS One 2015;10(2):e0116820 [FREE Full text] [doi: 10.1371/journal.pone.0116820] [Medline: 25658103]

2. Fleury M, Grenier G, Bamvita J, Perreault M, Kestens Y, Caron J. Comprehensive determinants of health service utilisation for mental health reasons in a Canadian catchment area. Int J Equity Health 2012 Apr 2;11:20 [FREE Full text] [doi: 10.1186/1475-9276-11-20] [Medline: 22469459]

3. Mojtabai R, Olfson M, Sampson NA, Jin R, Druss B, Wang PS, et al. Barriers to mental health treatment: results from the National Comorbidity Survey Replication. Psychol Med 2011 Aug;41(8):1751-1761 [FREE Full text] [doi: 10.1017/S0033291710002291] [Medline: 21134315]

4. Vasiliadis H, Lesage A, Adair C, Boyer R. Service use for mental health reasons: cross-provincial differences in rates, determinants, and equity of access. Can J Psychiatry 2005 Sep;50(10):614-619. [doi: 10.1177/070674370505001007] [Medline: 16276852]

5. Marzano L, Bardill A, Fields B, Herd K, Veale D, Grey N, et al. The application of mHealth to mental health: opportunities and challenges. Lancet Psychiatry 2015 Oct;2(10):942-948. [doi: 10.1016/S2215-0366(15)00268-0] [Medline: 26462228]

6. Cunningham JA, Gulliver A, Farrer L, Bennett K, Carron-Arthur B. Internet interventions for mental health and addictions: current findings and future directions. Curr Psychiatry Rep 2014 Dec;16(12):521. [doi: 10.1007/s11920-014-0521-5] [Medline: 25308390]

7. Firth J, Torous J, Nicholas J, Carney R, Rosenbaum S, Sarris J. Can smartphone mental health interventions reduce symptoms of anxiety? A meta-analysis of randomized controlled trials. J Affect Disord 2017 Aug 15;218:15-22 [FREE Full text] [doi: 10.1016/j.jad.2017.04.046] [Medline: 28456072] 
8. Firth J, Torous J, Nicholas J, Carney R, Pratap A, Rosenbaum S, et al. The efficacy of smartphone-based mental health interventions for depressive symptoms: a meta-analysis of randomized controlled trials. World Psychiatry 2017 Oct;16(3):287-298 [FREE Full text] [doi: 10.1002/wps.20472] [Medline: 28941113]

9. Donkin L, Christensen H, Naismith SL, Neal B, Hickie IB, Glozier N. A systematic review of the impact of adherence on the effectiveness of e-therapies. J Med Internet Res 2011;13(3):e52 [FREE Full text] [doi: 10.2196/jmir.1772] [Medline: 21821503]

10. Christensen H, Griffiths KM, Farrer L. Adherence in internet interventions for anxiety and depression. J Med Internet Res 2009;11(2):e13 [FREE Full text] [doi: 10.2196/jmir.1194] [Medline: 19403466]

11. Greenhalgh T, Shaw S, Wherton J, Hughes G, Lynch J, A'Court C, et al. SCALS: a fourth-generation study of assisted living technologies in their organisational, social, political and policy context. BMJ Open 2016 Feb 15;6(2):e010208 [FREE Full text] [doi: 10.1136/bmjopen-2015-010208] [Medline: 26880671]

12. Batterham PJ, Sunderland M, Calear AL, Davey CG, Christensen H, Teesson M, et al. Developing a roadmap for the translation of e-mental health services for depression. Aust N Z J Psychiatry 2015 Apr 23:776-784. [doi: 10.1177/0004867415582054] [Medline: 25907269]

13. Michie S, Yardley L, West R, Patrick K, Greaves F. Developing and evaluating digital interventions to promote behavior change in health and health care: recommendations resulting from an international workshop. J Med Internet Res 2017 Jun 29;19(6):e232 [FREE Full text] [doi: 10.2196/jmir.7126] [Medline: 28663162]

14. Naylor D, Girard F, Mintz J, Fraser N, Jenkins T, Power C. Health - Canada.ca. 2018. Unleashing innovationxcellent healthcare for Canada - report of the advisory panel on healthcare innovation URL: https://tinyurl.com/qx2cf8z [accessed 2019-05-13] [WebCite Cache ID 78LLlkldd]

15. Harding C, Chung H. Behavioral health support and online peer communities: international experiences. Mhealth 2016;2:43 [FREE Full text] [doi: 10.21037/mhealth.2016.10.04] [Medline: 28293613]

16. Hensel JM, Shaw J, Jeffs L, Ivers NM, Desveaux L, Cohen A, et al. A pragmatic randomized control trial and realist evaluation on the implementation and effectiveness of an internet application to support self-management among individuals seeking specialized mental health care: a study protocol. BMC Psychiatry 2016 Oct 18;16(1):350 [FREE Full text] [doi: 10.1186/s12888-016-1057-5] [Medline: 27756281]

17. Devilly GJ, Borkovec TD. Psychometric properties of the credibility/expectancy questionnaire. J Behav Ther Exp Psychiatry 2000 Jun;31(2):73-86. [Medline: 11132119]

18. Price M, Anderson P, Henrich CC, Rothbaum BO. Greater expectations: using hierarchical linear modeling to examine expectancy for treatment outcome as a predictor of treatment response. Behav Ther 2008 Dec;39(4):398-405 [FREE Full text] [doi: 10.1016/j.beth.2007.12.002] [Medline: 19027436 ]

19. Harris PA, Taylor R, Thielke R, Payne J, Gonzalez N, Conde JG. Research electronic data capture (REDCap)--a metadata-driven methodology and workflow process for providing translational research informatics support. J Biomed Inform 2009 Apr;42(2):377-381 [FREE Full text] [doi: 10.1016/j.jbi.2008.08.010] [Medline: 18929686]

20. Salzer MS, Brusilovskiy E. Advancing recovery science: reliability and validity properties of the Recovery Assessment Scale. Psychiatr Serv 2014 Apr 1;65(4):442-453. [doi: 10.1176/appi.ps.201300089] [Medline: 24487405]

21. Willer B, Ottenbacher KJ, Coad ML. The community integration questionnaire. A comparative examination. Am J Phys Med Rehabil 1994 Apr;73(2):103-111. [Medline: $\underline{\text { 8148099] }}$

22. Löwe B, Unützer J, Callahan CM, Perkins AJ, Kroenke K. Monitoring depression treatment outcomes with the patient health questionnaire-9. Med Care 2004 Dec;42(12):1194-1201. [Medline: 15550799]

23. Euroqol. 2018. Valuation of EQ-5D URL: https://euroqol.org/eq-5d-instruments/valuation-of-eq-5d/ [accessed 2019-05-14] [WebCite Cache ID 78NX02aCi]

24. Stålnacke B. Post-traumatic stress, depression, and community integration a long time after whiplash injury. Ment Illn 2010 Jan 25;2(1):e4 [FREE Full text] [doi: 10.4081/mi.2010.e4] [Medline: 25478087]

25. Gilbody S, Littlewood E, Hewitt C, Brierley G, Tharmanathan P, Araya R, et al. Computerised cognitive behaviour therapy (cCBT) as treatment for depression in primary care (REEACT trial): large scale pragmatic randomised controlled trial. $\mathrm{Br}$ Med J 2015;351:h5627 [FREE Full text] [Medline: 26559241]

26. Robertson TM, Lofgren RP. Where population health misses the mark: breaking the 80/20 rule. Acad Med 2015 Mar;90(3):277-278. [doi: 10.1097/ACM.0000000000000627] [Medline: 25551859]

27. Griffiths KM, Calear AL, Banfield M. Systematic review on Internet Support Groups (ISGs) and depression (1): do ISGs reduce depressive symptoms? J Med Internet Res 2009 Sep 30;11(3):e40 [FREE Full text] [doi: 10.2196/jmir.1270] [Medline: 19793719]

28. Eysenbach G, Powell J, Englesakis M, Rizo C, Stern A. Health related virtual communities and electronic support groups: systematic review of the effects of online peer to peer interactions. Br Med J 2004 May 15;328(7449):1166 [FREE Full text] [doi: 10.1136/bmj.328.7449.1166] [Medline: 15142921$]$

29. Takahashi Y, Uchida C, Miyaki K, Sakai M, Shimbo T, Nakayama T. Potential benefits and harms of a peer support social network service on the internet for people with depressive tendencies: qualitative content analysis and social network analysis. J Med Internet Res 2009 Jul 23;11(3):e29 [FREE Full text] [doi: 10.2196/jmir.1142] [Medline: 19632979] 
30. Anguera JA, Jordan JT, Castaneda D, Gazzaley A, Areán PA. Conducting a fully mobile and randomised clinical trial for depression: access, engagement and expense. BMJ Innov 2016 Jan;2(1):14-21 [FREE Full text] [doi: 10.1136/bmjinnov-2015-000098] [Medline: 27019745]

31. Strand M, Gammon D, Ruland CM. Transitions from biomedical to recovery-oriented practices in mental health: a scoping review to explore the role of Internet-based interventions. BMC Health Serv Res 2017 Dec 7;17(1):257 [FREE Full text] [doi: 10.1186/s12913-017-2176-5] [Medline: 28388907]

32. Barratt H, Shaw J, Simpson L, Bhatia S, Fulop N. Health services research: building capacity to meet the needs of the health care system. J Health Serv Res Policy 2017 Dec;22(4):243-249 [FREE Full text] [doi: 10.1177/1355819617714815] [Medline: 28786700 ]

\author{
Abbreviations \\ BWW: Big White Wall \\ CIQ: Community Integration Questionnaire \\ DTG: delayed treatment group \\ EQ-5D-5L: EuroQOL 5-dimension quality of life questionnaire \\ GAD-7: Generalized Anxiety Disorder Questionnaire-7 item \\ ITG: immediate treatment group \\ PHQ-9: Patient Health Questionnaire-9 item \\ RAS-r: Recovery Assessment Scale, revised \\ VAS: visual analog scale
}

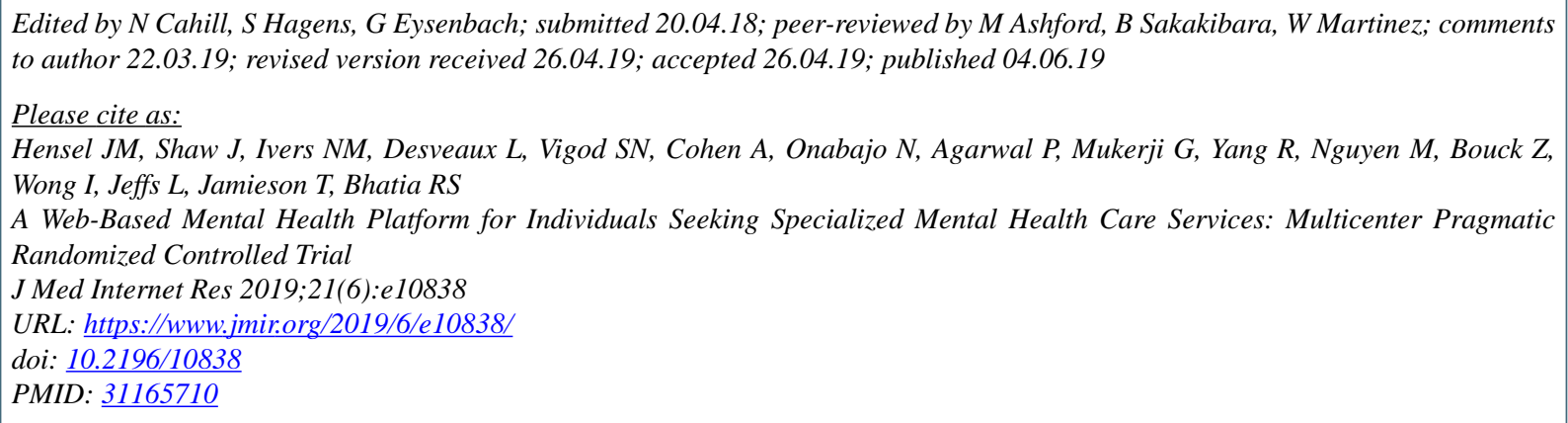

(CJennifer M Hensel, James Shaw, Noah M Ivers, Laura Desveaux, Simone N Vigod, Ashley Cohen, Nike Onabajo, Payal Agarwal, Geetha Mukerji, Rebecca Yang, Megan Nguyen, Zachary Bouck, Ivy Wong, Lianne Jeffs, Trevor Jamieson, R Sacha Bhatia. Originally published in the Journal of Medical Internet Research (http://www.jmir.org), 04.06.2019. This is an open-access article distributed under the terms of the Creative Commons Attribution License (https://creativecommons.org/licenses/by/4.0/), which permits unrestricted use, distribution, and reproduction in any medium, provided the original work, first published in the Journal of Medical Internet Research, is properly cited. The complete bibliographic information, a link to the original publication on http://www.jmir.org/, as well as this copyright and license information must be included. 